City University of New York (CUNY) CUNY Academic Works

6-2014

\title{
The Asymptotic Dirichlet Problems on manifolds with unbounded negative curvature
}

Ran Ji

Graduate Center, City University of New York

\section{How does access to this work benefit you? Let us know!}

More information about this work at: https://academicworks.cuny.edu/gc_etds/232

Discover additional works at: https://academicworks.cuny.edu

This work is made publicly available by the City University of New York (CUNY).

Contact: AcademicWorks@cuny.edu 


\title{
The Asymptotic Dirichlet Problems on manifolds with unbounded negative curvature
}

\author{
by
}

Ran Ji

A dissertation submitted to the Graduate Faculty in Mathematics in partial fulfillment of the requirements for the degree of Doctor of Philosophy, The City University of New York. 
(C) 2014

Ran Ji

All Rights Reserved 
This manuscript has been read and accepted for the Graduate Faculty in Mathematics in satisfaction of the dissertation requirements for the degree of Doctor of Philosophy.

Józef Dodziuk

Date

Chair of Examining Committee

Linda Keen

Date

Executive Officer

Józef Dodziuk

Zeno Huang

Marcello Lucia

Supervisory Committee 


\author{
Abstract \\ The Asymptotic Dirichlet Problems on \\ manifolds with unbounded negative curvature \\ by \\ Ran Ji
}

Advisor: Józef Dodziuk

Elton P. Hsu used probabilistic method to show that the asymptotic Dirichlet problem is uniquely solvable if the curvature satisfies the condition $-C e^{(2-\eta) r(x)} \leq K_{M}(x) \leq-1$ with $\eta>0$. We give an analytical proof of the same statement. In addition, using this new approach we are able to establish two boundary Harnack inequalities under the curvature condition $-C e^{(2 / 3-\eta) r(x)} \leq K_{M}(x) \leq-1$ with $\eta>0$. This implies that there is a natural homeomorphism between the Martin boundary and the geometric boundary of $M$. As far as we know, this is the first result of this kind under unbounded curvature conditions. Our proofs are modifications of arguments due to M. T. Anderson and R. Schoen. 


\section{Acknowledgements}

I would like to express the deepest appreciation to my advisor, Professor Józef Dodziuk, for his patience, encouragement and guidance without which I would never have been able to finish my dissertation. I would also like to thank Professor Zeno Huang and Professor Marcello Lucia for their useful comments, suggestions and their support in my job hunting. 


\section{Contents}

$\begin{array}{ll}\text { Introduction } & 1\end{array}$

1 Asymptotic Dirichlet Problems 5

1.1 Preliminaries .................... 5

1.2 Proof of Theorem $0.2 \ldots \ldots \ldots \ldots$

2 Martin Boundary and Boundary Harnack Inequalities 20

2.1 Martin Boundary . . . . . . . . . . . . . . . . 20

2.2 Proofs of two Boundary Harnack

Inequalities . . . . . . . . . . . . . . . . 23

$\begin{array}{ll}\text { Bibliography } & 40\end{array}$ 


\section{Introduction}

In this paper we discuss the solvability of the asymptotic Dirichlet problem and the equivalence of the geometric and Martin boundary on manifolds with negative curvature.

Let $M$ be a complete, simply connected n-dimensional Riemannian manifold whose sectional curvature is bounded from above by a negative constant. Fix a base point $p \in M$. It is well known that the exponential map $\exp _{p}: \mathrm{T}_{p} M \rightarrow M$ is a diffeomorphism. $S(\infty)$, which is defined as the set of equivalence classes of geodesic rays, can be identified with the unit sphere in $\mathrm{T}_{p}(M)$. A basic fact is that $\bar{M}=M \cup S(\infty)$ with the 'cone topology' is a compactification of $M$ [SY94].

Given $\varphi \in C^{0}(S(\infty))$, the asymptotic Dirichlet problem is to find a continuous function $f$ on $\bar{M}$ such that $f$ is harmonic on $M$ and $f=\varphi$ on $S(\infty)$. The case when $M$ has pinched curvature was solved in 1983 independently by Anderson [And83] and Sullivan [Sul83]. Anderson's approach was 
to construct appropriate convex sets and use the convexity property of Choi [Cho84]. A simpler proof was given by Anderson and Schoen [AS85] in 1985. In 1992, Borbély was able to replace the lower bound of the curvature by an unbounded growth function. His proof was based upon that of Anderson, namely he proved the following theorem.

Theorem 0.1. ([Bor92]) Let $M$ be a complete, simply connected Riemannian manifold with negative sectional curvature. Let $r=d(p, \cdot)$ denote the distance function and $\lambda<\frac{1}{3}$ be a positive constant. If the sectional curvature $K_{M}$ satisfies $K_{M}(x) \leq-1$ everywhere and $-e^{\lambda r(x)} \leq K_{M}(x)$ outside a compact subset of $M$, then the asymptotic Dirichlet problem is uniquely solvable.

Hsu was able to get a better lower bound of the curvature condition using a probabilistic method. His result is as follows.

Theorem 0.2. ([Hsu03]) Let $M$ be a complete, simply connected Riemannian manifold whose sectional curvature $K_{M}$ satisfies $-C e^{\lambda r(x)} \leq K_{M}(x) \leq$ -1 on $M$ for some $\lambda<2$. Then the asymptotic Dirichlet problem is uniquely solvable.

We will give an analytical proof of Theorem 0.2 in Chapter 1 based upon that of Anderson and Schoen [AS85]. A key refinement is that instead of taking the average $\bar{\varphi}$ of the extended function $\varphi$ in a ball of fixed radius, 
we let the radius vary. Then with the help of Bishop volume comparison theorem, we can show that even under relaxed curvature growth condition, the argument still works and yields Hsu's result.

On a non-parabolic manifold, i.e., a manifold that possesses positive a Green's function, one can define the Martin boundary which describes the behavior of harmonic functions at infinity. We will give more details in Section 2.1. A natural question is whether the Martin boundary is the same as the geometric boundary. Anderson and Schoen showed that we can identify them when the manifold has pinched negative curvature.

Theorem 0.3. ([AS85]) Let $M$ be a complete, simply connected Riemannian manifold whose sectional curvature satisfies $-b^{2} \leq K_{M} \leq-a^{2}<0$. Then there exists a natural homeomorphism $\Phi: \mathcal{M} \rightarrow S(\infty)$ from the the Martin boundary $\mathcal{M}$ of $M$ to the geometric boundary $S(\infty)$. Moreover, $\Phi^{-1}$ is Hölder continuous.

To prove Theorem 0.3, they established two boundary Harnack inequalities, which estimate the growth of positive harmonic functions in cones which vanish continuously at infinity. In Section 2.2, we relax the curvature assumption in Theorem 0.3 and establish the Harnack inequalities. It follows that the Martin boundary can be identified with the geometric boundary. To be 
precise, we prove the following theorem.

Theorem 0.4. Let $M$ be a complete, simply connected Riemannian manifold whose sectional curvature $K_{M}$ satisfies $-C e^{\lambda r(x)} \leq K_{M}(x) \leq-1$ on $M$ for some $\lambda<\frac{2}{3}$. Then there is a natural homeomorphism between the geometric boundary and Martin boundary of $M$.

Our result on the Martin boundary is the first one that allows the sectional curvature go to $-\infty$ as $r \rightarrow \infty$.

Remark 0.1 Theorem 0.2 holds for $\lambda<2$. However, we were able to prove Theorem 0.4 only under the stronger condition $\lambda<\frac{2}{3}$. This is because Theorem 0.4 follows from the boundary Harnack inequalities in Section 2.2, which we can prove only under the stronger curvature condition. It is possible that the boundary Harnack inequalities and therefore Theorem 0.4 remain true under the relaxed curvature condition, $\lambda<2$. 


\section{Chapter 1}

\section{Asymptotic Dirichlet Problems}

\section{$1.1 \quad$ Preliminaries}

Throughout this section we assume that $M$ is a complete, simply connected Riemannian manifold of $\mathrm{n}$ dimensions with sectional curvature $K_{M}(x) \leq-1$.

Denote by $H(-1)$ the two-dimensional hyperbolic plane with constant curvature -1 . We have the following well known Toponogov comparison theorem [SY94].

Theorem 1.1. Let $\triangle p x y$ be a geodesic triangle in $M$ with vertices $p, x, y$. Suppose $\triangle \tilde{p} \tilde{x} \tilde{y}$ is the corresponding geodesic triangle in $H(-1)$, such that the corresponding sides have the same length. Then we have

$$
\angle(p x, p y) \leq \angle(\tilde{p} \tilde{x}, \tilde{p} \tilde{y})
$$

where $\angle(p x, p y)$ denotes the angle at $p$ between the geodesic segments $p x$ and 
py.

In this proof we assume that all geodesics are parameterized by arc length.

Two geodesic rays $\gamma_{1}$ and $\gamma_{2}$ are said to be equivalent, denoted by $\gamma_{1} \sim \gamma_{2}$ if there exists a constant $C$ such that for any $t \geq 0$ we have

$$
d\left(\gamma_{1}(t), \gamma_{2}(t)\right) \leq C
$$

Define $S(\infty)$, the sphere at infinity, to be

$$
S(\infty)=\text { the set of all geodesic rays } / \sim \text {. }
$$

Let $S_{p}$ denote the unit sphere in $T_{p}(M)$. Given $\omega \in S_{p}$, there exists a unique geodesic ray $\gamma:[0, \infty) \rightarrow M$ satisfying $\gamma(0)=p$ and $\gamma^{\prime}(0)=\omega$. Two geodesic rays $\gamma_{1}$ and $\gamma_{2}$ starting from $p$ are equivalent if and only if $\gamma_{1}=\gamma_{2}$. At the same time each equivalence class contains a representative emanating from $p$. Thus $S(\infty)$ can be identified with $S_{p}$ for each $p \in M$.

Now we can define the cone $C_{p}(\omega, \delta)$ around $\omega$ of angle $\delta$ by

$$
C_{p}(\omega, \delta)=\left\{x \in M: \angle\left(\omega, \gamma_{p x}^{\prime}(0)\right)<\delta\right\}
$$

where $\gamma_{p x}$ denotes the geodesic ray starting from $p$ that passes through $x$. We call

$$
T_{p}(\omega, \delta, R)=C_{p}(\omega, \delta) \backslash B_{p}(R)
$$


a truncated cone of radius $R$. We denote $M \cup S(\infty)$ by $\bar{M}$. Then the set of $T_{p}(\omega, \delta, R)$ for all $\omega \in S_{p}, \delta$ and $R>0$ and $B_{q}(r)$ for all $q \in M$ and $r>0$ form a basis of a topology on $\bar{M}$, which is called the cone topology. This topology makes $\bar{M}$ a compactification of $M$ [SY94].

Remark 1.1 The cone topology on $\bar{M}$ is independent of the choice of $p$.

Remark 1.2 Anderson and Schoen showed that if $-b^{2} \leq K_{M} \leq-a^{2}<0$, then the topological structure is $C^{\alpha}$, where $\alpha=a / b$.

From now on we identify $S(\infty)$ with $S_{p}$ and its image under the exponential map $\exp _{p}\left(S_{p}\right)$. Let $(r, \theta)$ be the normal polar coordinates at $p$. Then $\varphi \in C^{0}(S(\infty))$ can be written as $\varphi=\varphi(\theta)$. Assume that Theorem 0.2 is true for all $\varphi \in C^{\infty}\left(S_{p}\right)$. Given $\varphi \in C^{0}\left(S_{p}\right)$, let $\varphi_{n} \in C^{\infty}\left(S_{p}\right)$ be a sequence of functions such that $\varphi_{n} \rightarrow \varphi$ uniformly. Then there exists a sequence of harmonic functions $u_{n} \in C^{\infty}(M) \cap C^{0}(\bar{M})$ satisfying $u_{n}(r, \theta) \rightarrow \varphi_{n}(\theta)$ as $r \rightarrow \infty$. By the maximum principle $u_{n} \rightarrow u$ uniformly on $\bar{M}$ and $\left.u\right|_{S(\infty)}=\varphi$. This shows that without loss of generality, we may assume that $\varphi \in C^{\infty}\left(S_{p}\right)$.

Extend $\varphi$ to $M \backslash\{p\}$ by defining

$$
\varphi(r, \theta)=\varphi(\theta)
$$

for $r>0$. We still use the letter $\varphi$ to denote the extended function. Then $\varphi$ 
is smooth and bounded on $M \backslash\{p\}$.

Let

$$
\operatorname{Osc}_{B_{x}(d)} \varphi=\sup _{y \in B_{x}(d)}|\varphi(y)-\varphi(x)|
$$

be the oscillation of $\varphi$ in the geodesic ball $B_{x}(d)$.

Since $\varphi \in C^{\infty}\left(S_{p}\right)$, it is Lipschitz continuous on $S_{p}$. We have for $y \in$ $B_{x}(d)$

$$
|\varphi(y)-\varphi(x)|=\left|\varphi\left(\theta^{\prime}\right)-\varphi(\theta)\right| \leq C\left|\theta^{\prime}-\theta\right|=C \angle(p x, p y),
$$

where $\theta, \theta^{\prime}$ are the spherical coordinates of $x$ and $y$ respectively.

Now it is necessary to estimate the angle $\angle(p x, p y)$.

Lemma 1.2. Let $M$ be a complete, simply connected Riemannian manifold with sectional curvature $K_{M} \leq-1$, and let $p, x, y$ be three points in $M$. Suppose that $d(p, x)=s$, and $y \in B_{x}(d)$ with $d<s$. We have

$$
\angle(p x, p y)<\frac{2 d}{e^{s-d}-1}
$$

The proof is based on a computation in the hyperbolic plane and the Topogonov comparison theorem. This Lemma is similar to that in [Bor92]. For completeness, we include the proof here.

Let $\triangle \tilde{p} \tilde{x} \tilde{y}$ be the corresponding geodesic triangle in $H(-1)$ such that $d(\tilde{p}, \tilde{x})=d(p, x)=s, d(\tilde{x}, \tilde{y})=d(x, y)=d^{\prime}<d<s$ and $d(\tilde{p}, \tilde{y})=d(p, y)$. 
We use the Poincare disk model to compute $\angle(\tilde{p} \tilde{x}, \tilde{p} \tilde{y})$ in the unit Euclidean ball $B^{2}$ with metric

$$
\mathrm{d} s_{H}^{2}=4 \frac{d r^{2}+r^{2} d \phi^{2}}{\left(1-r^{2}\right)^{2}}
$$

where $(r, \phi)$ are the polar coordinates of $B^{2}$.

Without loss of generality, we may assume that $\tilde{p}$ is the center of $B^{n}$. Let $\tilde{\tilde{x}}$ be the intersection of the geodesic sphere $S_{\tilde{x}}(d)$ and the line segment $\tilde{p} \tilde{x}$. Then $d_{H}(\tilde{p}, \tilde{\tilde{x}})=s-d$. From (1.2) we can easily compute the Euclidean distance between $\tilde{p}$ and $\tilde{x}$ :

$$
d_{E}(\tilde{p}, \tilde{\tilde{x}})=\frac{e^{s-d}-1}{e^{s-d}+1}
$$

Let $\tilde{\tilde{y}}$ be the intersection of the geodesic sphere $S_{\tilde{p}}(s-d)$ and the line segment $\tilde{p} \tilde{y}$. Denote by $\operatorname{arc}(\tilde{\tilde{x}}, \tilde{\tilde{y}})$ the circular arc joining $\tilde{\tilde{x}}$ and $\tilde{\tilde{y}}, l_{E}$ and $l_{H}$ the lengths of curves in Euclidean and hyperbolic metrics respectively. We have $l_{H}(\operatorname{arc}(\tilde{\tilde{x}}, \tilde{\tilde{y}}))<d_{H}(\tilde{x}, \tilde{y})=d^{\prime}$. In fact, let $\gamma_{1}(\phi)=\left(d_{E}(\tilde{p}, \tilde{\tilde{x}}), \phi\right)$ and $\gamma_{2}(\phi)=(r(\phi), \phi)$ be the parameterization of $\operatorname{arc}(\tilde{\tilde{x}}, \tilde{\tilde{y}})$ and the geodesic segment $\tilde{x} \tilde{y}$ respectively. We have

$$
\left|\gamma_{1}^{\prime}(\phi)\right|=\frac{2 d_{E}(\tilde{p}, \tilde{\tilde{x}})}{1-\left(d_{E}(\tilde{p}, \tilde{\tilde{x}})\right)^{2}}
$$

Also

$$
\left|\gamma_{2}^{\prime}(\phi)\right|=2 \frac{\sqrt{r^{\prime 2}(\phi)+r^{2}(\phi)}}{1-r^{2}(\phi)} \geq \frac{2 r(\phi)}{1-r^{2}(\phi)} .
$$


We have $r(\phi)>d_{E}(\tilde{p}, \tilde{\tilde{x}})$ for all $\phi$ since the geodesic ball $B_{\tilde{x}}\left(d^{\prime}\right)$ lies completely outside $B_{\tilde{p}}(s-d)$, which implies $\left|\gamma_{2}^{\prime}(\phi)\right|>\left|\gamma_{1}^{\prime}(\phi)\right|$ and thus $l_{H}(\operatorname{arc}(\tilde{\tilde{x}}, \tilde{\tilde{y}}))<d(\tilde{x}, \tilde{y})=d^{\prime}<d$. By $(1.2)$ again we have

$$
\begin{aligned}
l_{E}(\operatorname{arc}(\tilde{\tilde{x}}, \tilde{\tilde{y}})) & \leq \frac{1}{2} \cdot\left(1-\left(d_{E}(\tilde{p}, \tilde{\tilde{x}})\right)^{2}\right) \cdot l_{H}(\operatorname{arc}(\tilde{\tilde{x}}, \tilde{\tilde{y}})) \\
& <\frac{d}{2} \cdot\left(1-\left(d_{E}(\tilde{p}, \tilde{\tilde{x}})\right)^{2}\right)
\end{aligned}
$$

Then

$$
\begin{aligned}
\angle(\tilde{p} \tilde{x}, \tilde{p} \tilde{y})=\angle(\tilde{p} \tilde{\tilde{x}}, \tilde{p} \tilde{\tilde{y}}) & =\frac{l_{E}(\operatorname{arc}(\tilde{\tilde{x}}, \tilde{\tilde{y}}))}{d_{E}(\tilde{p}, \tilde{\tilde{x}})} \\
& <\frac{d}{2} \cdot \frac{1-\left(d_{E}(\tilde{p}, \tilde{\tilde{x}})\right)^{2}}{d_{E}(\tilde{p}, \tilde{\tilde{x}})} \\
& <\frac{2 d}{e^{s-d}-1} .
\end{aligned}
$$

By Theorem 1.1 we have $\angle(p x, p y) \leq \angle(\tilde{p} \tilde{x}, \tilde{p} \tilde{y})<\frac{2 d}{e^{s-d}-1}$. Lemma 1.2 is proved.

\subsection{Proof of Theorem 0.2}

Throughout this section we assume that $M$ is a complete, simply connected n-dimensional Riemannian manifold with sectional curvature bounded from above by -1 and satisfies

$$
-C e^{(2-2 \delta) r(x)} \leq K_{M}(x)
$$


outside a compact subset of $M$ for some $\delta>0$.

Remark 1.3 Without loss of generality, we may assume that the condition $-C e^{(2-2 \delta) r(x)} \leq K_{M}(x) \leq-1$ holds for some large enough constant $C$ on the whole manifold.

Remark 1.4 The factor 2 before $\delta$ is just for notational convenience.

We follow Anderson and Schoen's argument.

Let

$$
d(x)=e^{-(1-\delta) r(x)}
$$

We estimate the oscillation of $\varphi$ in the geodesic ball $B_{x}(d(x))$. Combining equation (1.1) and Lemma 1.2 we see easily that

$$
\operatorname{osc}_{B_{x}(d(x))} \varphi=\mathrm{O}\left(e^{-(2-\delta) r(x))}\right)
$$

Now we take the average $\bar{\varphi}$ of $\varphi$ in the ball $B_{x}(d(x))$ in the following way. Let $\chi \in C_{0}^{\infty}(\mathbb{R})$ be a function satisfying $0 \leq \chi \leq 1, \chi(t)=0$ for $|t| \geq 1$ and $\chi(t)=1$ for $|t| \leq 1 / 4$. Let

$$
u(x, y)=\chi\left(e^{(2(1-\delta) r(x)} \rho_{x}^{2}(y)\right)
$$


where $\rho_{x}=d(x, \cdot)$. We have

$$
u(x, y)= \begin{cases}1 & \text { if } y \in \overline{B_{x}(d(x) / 2)} \\ 0 & \text { if } y \in M \backslash B_{x}(d(x)) .\end{cases}
$$

Now define

$$
\bar{\varphi}(x)=\frac{\int_{M} u(x, y) \varphi(y) d y}{\int_{M} u(x, y) d y}
$$

Since $\varphi$ is continuous and bounded on $M \backslash\{p\}, \bar{\varphi}$ is smooth on $M$. Then we have

$$
\begin{aligned}
|\bar{\varphi}(x)-\varphi(x)| & =\frac{\int_{B_{x}(d(x))} u(x, y)(\varphi(y)-\varphi(x)) d y}{\int_{B_{x}(d(x))} u(x, y) d y} \\
& \leq \sup _{y \in B_{x}(d(x))}|\varphi(y)-\varphi(x)| \\
& =\operatorname{osc}_{B_{x}(d(x))} \varphi \\
& =\mathrm{O}\left(e^{-(2-\delta) r(x))}\right),
\end{aligned}
$$

which implies $\bar{\varphi}$ and $\varphi$ have the same value on $S(\infty)$.

Let

$$
v(x)=\int_{M} u(x, y) d y
$$

it follows from $(2)$ that $\operatorname{Vol}\left(B_{x}(d(x) / 2)\right) \leq v(x) \leq \operatorname{Vol}\left(B_{x}(d(x))\right)$.

In the following we will simply write $d$ for $d(x), u$ for $u(x, y), \rho$ for $\rho_{x}(y)$ and $v$ for $v(x)$ and the operations $\nabla$ and $\Delta$ will always be with respect to $x$. 
We have

$$
\begin{aligned}
\Delta \bar{\varphi}\left(x_{0}\right) & =\left.\Delta\left(\bar{\varphi}(x)-\varphi\left(x_{0}\right)\right)\right|_{x=x_{0}} \\
& =\left.\int_{M} \Delta\left(\frac{u}{v}\right)\left(\varphi(y)-\varphi\left(x_{0}\right)\right) d y\right|_{x=x_{0}} .
\end{aligned}
$$

Direct computation gives

$$
\Delta\left(\frac{u}{v}\right)=\frac{v \Delta u-2 \nabla u \cdot \nabla v-u \Delta v}{v^{2}}+\frac{2 u}{v^{3}}|\nabla v|^{2} .
$$

Since $r$ and $\rho$ are both distance functions, we have $|\nabla r|=|\nabla \rho|=1$. Together with the fact that $\operatorname{supp} u \subset \overline{B_{x}(d(x))}$, we have

$$
\begin{aligned}
\nabla u & =\chi^{\prime}\left(e^{(2(1-\delta) r(x)} \rho_{x}^{2}(y)\right) \cdot\left(e^{2(1-\delta) r}\left((2-2 \delta) \rho^{2} \nabla r+2 \rho \nabla \rho\right)\right) \\
& =\mathrm{O}\left(e^{(1-\delta) r}\right)
\end{aligned}
$$

here we used $\rho=\mathrm{O}\left(e^{-(1-\delta) r}\right)$.

$$
\begin{aligned}
\Delta u= & \chi^{\prime \prime}\left(e^{(2(1-\delta) r(x)} \rho_{x}^{2}(y)\right) \cdot\left(e^{2(1-\delta) r}\left((2-2 \delta) \rho^{2} \nabla r+2 \rho \nabla \rho\right)\right)^{2} \\
& +\chi^{\prime}\left(e^{(2(1-\delta) r(x)}\right) \cdot\left(e ^ { 2 ( 1 - \delta ) r } \left((2-2 \delta)^{2} \rho^{2}|\nabla r|^{2}+4(2-2 \delta) \rho \nabla r \cdot \nabla \rho\right.\right. \\
& \left.\left.+(2-2 \delta) \rho^{2} \Delta r+2|\nabla \rho|^{2}+2 \rho \Delta \rho\right)\right) .
\end{aligned}
$$

We need the following Hessian comparison theorem from [SY94] to estimate $\Delta r$ and $\Delta \rho$. 
Theorem 1.3. Let $M_{1}$ and $M_{2}$ be two n-dimensional complete Riemannian manifolds. Assume that $\gamma_{i}:[0, a] \rightarrow M_{i}(i=1,2)$ are two geodesics parametrized by arc length, and $\gamma_{i}$ does not intersect the cut locus of $\gamma_{i}(0)$ for $i=1,2$. Let $r_{i}$ be the distance function from $\gamma_{i}(0)$ on $M_{i}$ and let $K_{i}$ be the sectional curvature of $M_{i}$. Assume that at $\gamma_{1}(t)$ and $\gamma_{2}(t), 0 \leq t \leq a$, we have

$$
K_{1}\left(X_{1}, \frac{\partial}{\partial \gamma_{1}}\right) \geq K_{2}\left(X_{2}, \frac{\partial}{\partial \gamma_{2}}\right)
$$

where $X_{i}$ is any unit vector in $T_{\gamma_{i}(t)} M_{i}$ perpendicular to $\frac{\partial}{\partial \gamma_{i}}$. Denote by $H\left(r_{i}\right)$ the Hessian of $r_{i}$, then

$$
H\left(r_{1}\right)\left(X_{1}, X_{1}\right) \leq H\left(r_{2}\right)\left(X_{2}, X_{2}\right),
$$

where $X_{i} \in T_{\gamma_{i}(a)} M_{i}$ with $\left\langle X_{i}, \frac{\partial}{\partial \gamma_{i}}\right\rangle\left(\gamma_{i}(a)\right)=0$ and $\left|X_{i}\right|=1$.

Since $\Delta r$ is the trace of $H(r)$, we have the following corollary.

Corollary 1.4. Let $M$ be an $n$-dimensional complete Riemannian manifold. If the sectional curvature satisfies $-k^{2} \leq K_{M}(x) \leq-1$ in the geodesic ball $\overline{B_{p}(R)}$, then

$$
(n-1) \operatorname{coth} r \leq \Delta r \leq(n-1) k \operatorname{coth} k r
$$

for $r \leq R$. In addition,

$$
n-1 \leq \Delta r \leq(n-1)\left(k+\frac{1}{r}\right)
$$


for $r \leq R$.

Since $-C e^{(2-2 \delta) r(x)} \leq K_{M}(x) \leq-1$ in $B_{p}(r(x))$. By Corollary 1.4 we have $n-1 \leq \Delta r(x) \leq(n-1)\left(1+C^{1 / 2} e^{(1-\delta) r(x)}\right)$ for $r(x) \geq 1$. So

$$
\Delta r=\mathrm{O}\left(e^{(1-\delta) r}\right)
$$

Since $-C e^{(2-2 \delta)(r(x)+1)} \leq K_{M}(x) \leq-1$ for $x \in B_{x}(d(x)) \subset B_{p}(r(x)+1)$. By Corollary 1.4 again we have

$$
\rho \Delta \rho \leq(n-1)\left(1+C e^{(1-\delta)(r+1)} \rho\right)=\mathrm{O}(1)
$$

when $d(x) / 2 \leq \rho \leq d(x)$. Apply (1.9) and (1.10) in (1.8) and use the fact that supp $\Delta u \subset \overline{B_{x}(d(x))} \backslash B_{x}(d(x) / 2)$ and $|\nabla r|=|\nabla \rho|=1$ we see that

$$
\Delta u=\mathrm{O}\left(e^{2(1-\delta) r}\right)
$$

To estimate $\nabla v$ we have

$$
\begin{aligned}
|\nabla v| & =\left|\nabla \int_{M} u d y\right| \\
& \leq \int_{M}|\nabla u| d y \\
& =\int_{B_{x}(d(x))}|\nabla u| d y
\end{aligned}
$$

thus

$$
|\nabla v|=\operatorname{Vol}\left(B_{x}(d(x)) \cdot \mathrm{O}\left(e^{(1-\delta) r}\right)\right.
$$


We also have

$$
\begin{aligned}
|\Delta v| & =\left|\Delta \int_{M} u d y\right| \\
& \leq \int_{M}|\Delta u| d y \\
& =\int_{B_{x}(d(x))}|\Delta u| d y
\end{aligned}
$$

thus

$$
|\Delta v|=\operatorname{Vol}\left(B_{x}(d(x)) \cdot \mathrm{O}\left(e^{2(1-\delta) r}\right)\right.
$$

Combining (1.7), (1.11), (1.12) and (1.13), we have the following Lemma.

\section{Lemma 1.5.}

$$
\begin{aligned}
\Delta\left(\frac{u}{v}\right)= & \left(\frac{1}{\operatorname{Vol}\left(B_{x}(d / 2)\right)}+\frac{\operatorname{Vol}\left(B_{x}(d)\right)}{\left(\operatorname{Vol}\left(B_{x}(d / 2)\right)\right)^{2}}+\frac{\left(\operatorname{Vol}\left(B_{x}(d)\right)\right)^{2}}{\left(\operatorname{Vol}\left(B_{x}(d / 2)\right)\right)^{3}}\right) \\
& \cdot \mathrm{O}\left(e^{2(1-\delta) r}\right) .
\end{aligned}
$$

To estimate $\Delta \bar{\varphi}(x)$, we need the following corollary of Bishop volume comparison theorem [Pet06].

Corollary 1.6. Let $M$ be a complete Riemannian manifold, and $c>0$ a constant. If $K_{M}(x) \geq-c^{2} k^{2}$ on $B_{p}(1)$ for some $k \geq 1$. Then $\frac{\operatorname{Vol}\left(B_{p}\left(\frac{1}{k}\right)\right)}{\operatorname{Vol}\left(B_{p}\left(\frac{1}{2 k}\right)\right)} \leq$ $C_{n}$, where $C_{n}$ is a constant that depends only on the dimension of $M$ and $c$.

Proof. By Bishop Volume Comparison Theorem, the ratio $\frac{\operatorname{Vol}\left(B_{p}(R)\right)}{V\left(-c^{2} k^{2}, R\right)}$ is non-increasing in $R$ for $R \leq 1$, where $V\left(-c^{2} k^{2}, R\right)$ is the volume of the 
geodesic balls of radius $R$ in the space form of constant curvature $-c^{2} k^{2}$. Thus

$$
\frac{\operatorname{Vol}\left(B_{p}\left(\frac{1}{k}\right)\right)}{V\left(-c^{2} k^{2}, \frac{1}{k}\right)} \leq \frac{\operatorname{Vol}\left(B_{p}\left(\frac{1}{2 k}\right)\right)}{V\left(-c^{2} k^{2}, \frac{1}{2 k}\right)}
$$

which can be written as

$$
\frac{\operatorname{Vol}\left(B_{p}\left(\frac{1}{k}\right)\right)}{\operatorname{Vol}\left(B_{p}\left(\frac{1}{2 k}\right)\right)} \leq \frac{V\left(-c^{2} k^{2}, \frac{1}{k}\right)}{V\left(-c^{2} k^{2}, \frac{1}{2 k}\right)}
$$

In the hyperbolic space of constant curvature $-K^{2}$, the volume of a ball of radius $r$ is given by

$$
V\left(-K^{2}, r\right)=\Omega_{n}\left(\frac{1}{K}\right)^{n-1} \int_{0}^{r} \sinh ^{n-1}(K r) d r
$$

where $\Omega_{n}$ is the surface area of the unit sphere in $\mathbb{R}^{n}$.

Computing using (1.15)

$$
V\left(-c^{2} k^{2}, \frac{1}{k}\right)=\Omega_{n}\left(\frac{1}{c k}\right)^{n-1} \int_{0}^{\frac{1}{k}} \sinh ^{n-1}(c k r) d r=\Omega_{n}\left(\frac{1}{c k}\right)^{n} \int_{0}^{c} \sinh ^{n-1} r d r
$$

and

$$
V\left(-c^{2} k^{2}, \frac{1}{2 k}\right)=\Omega_{n}\left(\frac{1}{c k}\right)^{n-1} \int_{0}^{\frac{1}{2 k}} \sinh ^{n-1}(c k r) d r=\Omega_{n}\left(\frac{1}{c k}\right)^{n} \int_{0}^{c / 2} \sinh ^{n-1} r d r .
$$

Now we can take $C_{n}=\frac{V\left(-c^{2} k^{2}, \frac{1}{k}\right)}{V\left(-c^{2} k^{2}, \frac{1}{2 k}\right)}=\frac{\int_{0}^{c} \sinh ^{n-1} r d r}{\int_{0}^{c / 2} \sinh ^{n-1} r d r}$, which is a constant that depends only on $n$ and $c$. 
We are now ready to estimate $\Delta \bar{\varphi}(x)$.

$$
\begin{aligned}
|\Delta \bar{\varphi}(x)| & =\left|\int_{M} \Delta\left(\frac{u}{v}\right)(\varphi(y)-\varphi(x)) d y\right| \\
& \leq \int_{B_{x}(d(x))}\left|\Delta\left(\frac{u}{v}\right)\right| d y \cdot \operatorname{osc}_{B_{x}(d(x))} \varphi \\
& \leq \sup _{B_{x}(d(x))}\left\{\left|\Delta\left(\frac{u}{v}\right)\right|\right\} \cdot \operatorname{Vol}\left(B_{x}(d(x))\right) \cdot \operatorname{osc}_{B_{x}(d(x))} \varphi \\
& =\left(\frac{\operatorname{Vol}\left(B_{x}(d)\right)}{\operatorname{Vol}\left(B_{x}(d / 2)\right)}+\frac{\left(\operatorname{Vol}\left(B_{x}(d)\right)\right)^{2}}{\left(\operatorname{Vol}\left(B_{x}(d / 2)\right)\right)^{2}}+\frac{\left(\operatorname{Vol}\left(B_{x}(d)\right)\right)^{3}}{\left(\operatorname{Vol}\left(B_{x}(d / 2)\right)\right)^{3}}\right) \cdot \mathrm{O}\left(e^{-\delta r}\right) .
\end{aligned}
$$

Observe that $B_{x}(d(x)) \subset B_{p}(r(x)+1)$ and on $B_{p}(r(x))$,

$$
K_{M}(x) \geq-C e^{(2-2 \delta) r(x)}=-C\left(\frac{1}{d(x)}\right)^{2}
$$

By Corollary 1.6, we have

$$
\frac{\operatorname{Vol}\left(B_{x}(d(x))\right)}{\operatorname{Vol}\left(B_{x}(d(x) / 2)\right)}=\mathrm{O}(1)
$$

It follows that

$$
\Delta \bar{\varphi}=\mathrm{O}\left(e^{-\delta r}\right)
$$

Define $g(x)=e^{-\delta_{0} r(x)}$, where $\delta_{0}$ is a positive constant to be chosen later.

We have

$$
\Delta g=g \cdot\left(-\delta_{0} \Delta r+\delta_{0}^{2}|\nabla r|^{2}\right)
$$

Since $K_{M} \leq-1$ we have $\Delta r \geq n-1$, choose $\delta_{0}<\delta$ small enough such 
that

$$
-\delta_{0} \Delta r+\delta_{0}^{2}|\nabla r|^{2}<-(n-1) \delta_{0}+\delta_{0}^{2}
$$

is less that a negative constant.

Now, since $\Delta \bar{\varphi}=\mathrm{O}\left(e^{-\delta r}\right)=\mathrm{o}\left(e^{-\delta_{0} r}\right)=\mathrm{o}(g)$, there exists a constant $\alpha>0$ such that

$$
\Delta(\alpha g) \leq-|\Delta \bar{\varphi}|
$$

which implies that $\bar{\varphi}-\alpha g$ is subharmonic and $\bar{\varphi}+\alpha g$ is superharmonic. It follows from the classical Perron's method that there exists a harmonic function $f$ such that

$$
\bar{\varphi}-\alpha g \leq f \leq \bar{\varphi}+\alpha g
$$

Since $\bar{\varphi}$ and $\varphi$ have the same boundary value and $g=0$ on $S(\infty), f=\varphi$ on the boundary. This completes the proof of Theorem 0.2. 


\section{Chapter 2}

\section{Martin Boundary and Boundary Harnack Inequalities}

\subsection{Martin Boundary}

Throughout this section we still assume that $M$ is a complete, simply connected n-dimensional Riemannian manifold whose sectional curvature satisfies

$$
-C e^{(2-2 \delta) r(x)} \leq K_{M}(x) \leq-1
$$

From Theorem 0.2 we know there exists a nontrivial bounded harmonic function $f$ on $M$. This implies (cf. [SY94]) that $M$ possesses a positive symmetric Green's function $G(p, x)$. Moreover, if we denote by $G_{i}(p, x)$ the Green's function on $\Omega_{i}$ with Dirichlet boundary condition, where $\left\{\Omega_{i}, i=\right.$ $1,2, \cdots\}$ is a compact exhaustion of $M$, then $G_{i}$ converges uniformly to $G$ on compact subsets of $M \backslash\{p\}$. 
We have shown on page 19 that if $\alpha>0$ is sufficiently small, then

$$
\Delta\left(e^{-\alpha r}\right) \leq 0
$$

on $M$. Let

$$
C_{1}=\sup _{\partial B_{p}(1)} G(p, x) e^{\alpha r(x)}>0
$$

we have

$$
\begin{gathered}
G_{i}(p, x) \leq G(p, x) \leq C_{1} e^{-\alpha r(x)} \text { on } \partial B_{p}(1), \\
0=G_{i}(p, x)<C_{1} e^{-\alpha r(x)} \text { on } \partial \Omega_{i},
\end{gathered}
$$

and

$$
0=\Delta G_{i} \geq \Delta\left(C_{1} e^{-\alpha r}\right) \text { on } \Omega_{i} \backslash B_{p}(1) .
$$

It follows from the maximum principle that

$$
G_{i} \leq C_{1} e^{-\delta r} \text { on } \Omega_{i} \backslash B_{p}(1)
$$

Passing to the limit

$$
G(p, x) \leq C_{1} e^{-\delta r(x)} \text { on } M \backslash B_{p}(1)
$$

which implies that $G$ extends continuously to $\bar{M}$ with value 0 on $S(\infty)$.

For $x, y \in M$, let

$$
h_{y}(x)=\frac{G(x, y)}{G(p, y)}
$$


be the normalized Green's function with $h_{y}(p)=1$. A sequence $Y=\left\{y_{i}\right\}$ is called fundamental if $h_{y_{i}}$ converges to a positive harmonic function $h_{Y}$ on $M$. Two fundamental sequences $Y$ and $\bar{Y}$ are said to be equivalent if the corresponding limiting positive harmonic functions $h_{Y}$ and $h_{\bar{Y}}$ are the same.

Definition 2.1. The Martin boundary $\mathcal{M}$ of $M$ is the set of equivalence classes of non-convergent fundamental sequences.

Let $\widetilde{M}=M \cup \mathcal{M}$. For each $y \in M$, all sequences converging to $y$ form an equivalence class $[Y]$. On the other hand, two fundamental sequences that have different limit points in $M$ are not equivalent. Thus $\widetilde{M}$ can be identified with the set of equivalence classes of fundamental sequences. Define a metric $\rho$ on $\widetilde{M}$

$$
\rho\left([Y],\left[Y^{\prime}\right]\right)=\sup _{B_{p}(1)}\left|h_{Y}(x)-h_{Y^{\prime}}(x)\right|
$$

for $[Y],\left[Y^{\prime}\right] \in \widetilde{M}$. The topology induced by $\rho$ makes $\widetilde{M}$ a compactification of $M$.

It is known from [SY94] that if for all $\theta_{1}, \theta_{2}$ with $0<\theta_{2}<\theta_{1}<\pi / 4$, there exists a positive constant $\alpha$ depending only on $n, C, \delta, \theta_{1}$ and $\theta_{2}$, such that for any positive harmonic function $u \in C^{0}\left(\overline{C_{p}\left(\theta_{1}\right)}\right)$ which vanishes on $\overline{C_{p}\left(\theta_{1}\right)} \cap S(\infty)$, the Harnack inequality

$$
u(x) \leq C_{1} u\left(p^{\prime}\right) e^{-\alpha r(x)}
$$


holds on $T\left(\theta_{2}, 1\right)$, then there is a natural surjection $\Phi: \mathcal{M} \rightarrow S(\infty)$. In fact, let $\left\{y_{k}\right\}$ be a sequence of points converging to $\xi \in S(\infty)$. Since $h_{y_{k}}(p)=1$, by the classical Harnack inequality, $h_{y_{k}}$ converge along some subsequence to a positive harmonic function $P_{\xi}$ satisfying $P_{\xi}(p)=1$. Then the above Harnack inequality implies that $P_{\xi}$ is continuous on $\bar{M} \backslash\{\xi\}$ and vanishes on $S(\infty) \backslash\{\xi\}$. It follows that $P_{\xi} \neq P_{\tilde{\xi}}$ if $\xi \neq \tilde{\xi}$. Thus a fundamental sequence has a unique limit point. The map is then well defined and surjective.

Moreover, if for any positive harmonic functions $u, v \in C^{0}\left(\overline{C_{p}\left(\theta_{1}\right)}\right)$ which vanish on $\overline{C_{p}\left(\theta_{1}\right)} \cap S(\infty)$, we have, for all $x \in T\left(\theta_{2}, 1\right)$,

$$
\tilde{C}^{-1} \frac{u\left(p^{\prime}\right)}{v\left(p^{\prime}\right)} \leq \frac{u(x)}{v(x)} \leq \tilde{C} \frac{u\left(p^{\prime}\right)}{v\left(p^{\prime}\right)}
$$

then $\Phi$ defined above is one-to-one and therefore a homeomorphism. For further details, see Chapter II in [SY94].

\subsection{Proofs of two Boundary Harnack Inequalities}

In this section we prove (2.2) and (2.3) to establish homeomorphism between $\mathcal{M}$ and $S(\infty)$. We assume that $M$ is a complete, simply connected n-dimensional Riemannian manifold whose sectional curvature satisfies

$$
-C e^{(2 / 3-2 \delta) r} \leq K_{M} \leq-1,
$$


unless otherwise stated.

Given $\omega \in S_{p}$. Let $p^{\prime}=\exp _{p} \omega$. Recall that $C_{p}(\theta)=C_{p}(\omega, \theta)$ is the cone about $\omega$ of angle $\theta$ at $p$, and $T_{p}(\theta, R)=T_{p}(\omega, \theta, R)=C_{p}(\omega, \theta) \backslash B_{p}(R)$ is the truncated cone.

Let $0<\theta_{2}<\theta_{1}<\pi / 4$ and $\theta_{3}=\left(\theta_{1}+\theta_{2}\right) / 2$.

We want to prove the following two boundary Harnack inequalities.

Theorem 2.2. Let $u$ be a positive harmonic function on $C_{p}\left(\theta_{1}\right)$ which is continuous on $\overline{C_{p}\left(\theta_{1}\right)}$ and vanishes on $\overline{C_{p}\left(\theta_{1}\right)} \cap S(\infty)$. Then for all $x \in$ $T\left(\theta_{2}, 1\right)$

$$
u(x) \leq \tilde{C} e^{-\alpha r(x)} u\left(p^{\prime}\right)
$$

where $\tilde{C}$ and $\alpha$ depend only on $n, C, \delta, \theta_{1}$ and $\theta_{2}$.

Theorem 2.3. Let $u, v$ be two positive harmonic functions on $C_{p}\left(\theta_{1}\right)$ which are continuous on $\overline{C_{p}\left(\theta_{1}\right)}$ and which vanish on $\overline{C_{p}\left(\theta_{1}\right)} \cap S(\infty)$. Then for all $x \in T\left(\theta_{2}, 1\right)$,

$$
\tilde{C}^{-1} \frac{u\left(p^{\prime}\right)}{v\left(p^{\prime}\right)} \leq \frac{u(x)}{v(x)} \leq \tilde{C} \frac{u\left(p^{\prime}\right)}{v\left(p^{\prime}\right)}
$$

where $\tilde{C}$ depends only on $n, C, \delta, \theta_{1}$ and $\theta_{2}$.

First we need to construct a cut-off function with small second derivatives.

Lemma 2.4. Given two constants $\alpha$ and $\beta$, there exists $\varphi \in C^{\infty}(M)$ and a 
constant $R_{0}>0$ such that

$$
\left\{\begin{aligned}
\varphi & =\alpha \text { on } T\left(\theta_{2}, R_{0}\right) \\
\varphi & =\beta \text { on } \partial C_{p}\left(\theta_{1}\right) \backslash B_{p}\left(R_{0}\right) \\
|\nabla \varphi| & =\mathrm{O}\left(e^{-r}\right) \text { on } T\left(\theta_{2}, R_{0}\right) \\
|\Delta \varphi| & =\mathrm{O}\left(e^{-(2 / 3+\delta) r}\right) \text { on } T\left(\theta_{2}, R_{0}\right)
\end{aligned}\right.
$$

Proof. Let $\psi \in C^{\infty}([0, \pi])$ be a function satisfying $0 \leq \psi \leq 1, \psi(t)=0$ for $t \in\left[0, \theta_{2}+\epsilon\right]$ and $\psi(t)=1$ for $t \in\left[\theta_{1}-\epsilon, \theta_{1}+\epsilon\right]$, where $\epsilon<\left(\theta_{1}-\theta_{2}\right) / 2$ is a small positive constant. Set

$$
\tilde{\psi}(x)=\psi(\angle(p x, v))
$$

$\tilde{\psi}$ is smooth and bounded on $M \backslash\{p\}$. We take the average $\varphi$ of $\tilde{\psi}$ in the ball $B_{x}\left(e^{(-1 / 3+\delta) r(x)}\right)$ by defining

$$
\varphi(x)=\frac{\int_{M} \chi\left(e^{(2 / 3-2 \delta) r(x)} \rho_{x}^{2}(y)\right) \tilde{\psi}(y) d y}{\int_{M} \chi\left(e^{(2 / 3-2 \delta) r(x)} \rho_{x}^{2}(y)\right) d y},
$$

where $\chi \in C_{0}^{\infty}(\mathbb{R})$ is a cut-off function satisfying $0 \leq \chi \leq 1, \chi(t)=0$ for $|t| \geq 1$ and $\chi(t)=1$ for $|t| \leq \frac{1}{4}$. The proof that $\varphi$ is our desired cut-off function is very similar to that of Theorem 0.2 on page 18 .

We will need the following gradient estimate for positive harmonic functions due to Yau.

Theorem 2.5. ([Yaur5]) Let $N$ be a complete Riemannian manifold of di- 
mension n. Suppose that the Ricci curvature on $B_{p}(R)$ is bounded from below by $-(n-1) K$ for some constant $K \geq 0$. If $u$ is a positive harmonic function on $B_{p}(R)$, then for any $0<\epsilon<1$, we have, for all $x \in B_{\epsilon R}$,

$$
\frac{|\nabla u|}{u} \leq \tilde{C}\left(\frac{1}{R}+\sqrt{K}\right)
$$

where $\tilde{C}$ is a constant depending only on $n$ and $\epsilon$.

Applying Theorem 2.5 on our manifold $M$ we get the following corollary.

Corollary 2.6. Let $M$ be a complete, simply connected n-dimensional Riemannian manifold with $-C e^{(2 / 3-2 \delta) r} \leq K_{M} \leq-1$. If $u$ is a positive harmonic function on $M$, then

$$
\left|\frac{\nabla u}{u}\right|(x) \leq \tilde{C} e^{(1 / 3-\delta / 2) r(x)},
$$

where $\tilde{C}$ depends only on $n, C$ and $\delta$.

Proof. For every $x \in M$, let

$$
R=\frac{2 / 3-\delta}{2 / 3-2 \delta} \cdot r(x)=C_{1} r(x)
$$

with $C_{1}>1$. We have

$$
K_{M} \geq-C e^{(2 / 3-2 \delta) R}=-C e^{(2 / 3-\delta) r}
$$

on $B_{p}(R)$. 
Apply Theorem 2.5 with $\epsilon=1 / C_{1}$ to obtain

$$
\left|\frac{\nabla u}{u}\right| \leq \tilde{C} e^{(1 / 3-\delta / 2)}
$$

on $B_{p}\left(R / C_{1}\right)=B_{p}(r(x))$.

Lemma 2.7. Let $u$ be a positive harmonic function on $C_{p}\left(\theta_{3}\right)$ which is continuous on $\overline{C_{p}\left(\theta_{3}\right)}$ and which vanishes on $\overline{C_{p}\left(\theta_{3}\right)} \cap S(\infty)$. Then for all $x \in T\left(\theta_{2}, 1\right)$,

$$
u(x) \leq \tilde{C} e^{-\alpha r(x)} \sup _{\partial C_{p}\left(\theta_{3}\right)} u
$$

where $\alpha$ is a constant depending only on $n, C, \delta, \theta_{1}$ and $\theta_{2}$.

Proof. By lemma 2.4, there exists $\varphi \in C^{\infty}(M)$ and a constant $R_{1}>0$ such that

$$
\left\{\begin{aligned}
\varphi & =0 \text { on } T\left(\theta_{2}, R_{1}\right) \\
\varphi & =1 \text { on } \partial C_{p}\left(\theta_{3}\right) \backslash B_{p}\left(R_{1}\right) \\
|\nabla \varphi| & =\mathrm{O}\left(e^{-r}\right) \text { on } T\left(\theta_{3}, R_{1}\right) \\
|\Delta \varphi| & =\mathrm{O}\left(e^{-(2 / 3+\delta) r}\right) \text { on } T\left(\theta_{3}, R_{1}\right)
\end{aligned}\right.
$$

Consider $e^{-\alpha r}$, where $\alpha<\frac{2}{3}+\delta$ is sufficiently small. We have

$$
\begin{aligned}
\Delta e^{-\alpha r} & =e^{-\alpha r}\left(\alpha^{2}-\alpha \Delta r\right) \\
& \leq e^{-\alpha r}\left(\alpha^{2}-(n-1) \alpha\right)<0 .
\end{aligned}
$$

Since $\Delta \varphi=\mathrm{O}\left(e^{-(2 / 3+\delta) r}\right)$, we have $|\Delta \varphi|<-C_{1} \Delta e^{-\alpha r}$ on $T\left(\theta_{3}, R_{0}\right)$ for $C_{1}$ 
and $R_{0}$ sufficiently large.

Let $f=\varphi+\tilde{C} e^{-\alpha r}$. We have $\Delta f \leq 0$ if $\tilde{C}>C_{1}$. Also $f \geq \varphi=1$ on $\partial C_{p}\left(\theta_{3}\right) \backslash B_{p}\left(R_{0}\right)$ and $f \geq 1$ on $\overline{B_{p}\left(R_{0}\right)}$, provided $\tilde{C}$ is sufficiently large.

Now consider $\bar{u}=u / \sup _{\partial C_{p}\left(\theta_{3}\right)} u . \bar{u}$ is harmonic and $\bar{u} \leq 1$ on $\partial C_{p}\left(\theta_{3}\right)$. We have $\Delta(\bar{u}-f) \geq 0$ on $C_{p}\left(\theta_{3}\right)$ and $\bar{u}-f \leq 0$ on $\partial C_{p}\left(\theta_{3}\right)$. By the maximum principle, $\bar{u} \leq f$ on $C_{p}\left(\theta_{3}\right)$. In particular,

$$
u(x) \leq f(x) \sup _{\partial C_{p}\left(\theta_{3}\right)} u=\tilde{C} e^{-\alpha r} \sup _{\partial C_{p}\left(\theta_{3}\right)} u
$$

for all $x \in T\left(\theta_{2}, R_{0}\right)$.

We are now ready to prove Theorem 2.2. By Lemma 2.7, it is sufficient to show that harmonic functions satisfying the given conditions and $u\left(p^{\prime}\right)=1$ are uniformly bounded on $\partial C_{p}\left(\theta_{3}\right)$. In the following we will use $C_{1}, C_{2}, \ldots$, $\alpha_{1}, \alpha_{2}, \ldots$ and $R_{1}, R_{2}, \ldots$ to denote positive constants depending only on $n$, $C, \delta, \theta_{1}$ and $\theta_{2}$.

By lemma 2.4, there exists $\varphi \in C^{\infty}(M)$ with $\frac{2}{3} \leq \varphi \leq 1$ and a constant 
$R_{0}>0$ such that

$$
\left\{\begin{aligned}
\varphi & =\frac{2}{3} \text { on } T\left(\theta_{3}, R_{0}\right), \\
\varphi & =1 \text { on } \partial C_{p}\left(\theta_{1}\right) \backslash B_{p}\left(R_{0}\right), \\
|\nabla \varphi| & =\mathrm{O}\left(e^{-r}\right) \text { on } T\left(\theta_{1}, R_{0}\right), \\
|\Delta \varphi| & =\mathrm{O}\left(e^{(-2 / 3-\delta) r}\right) \text { on } T\left(\theta_{1}, R_{0}\right) .
\end{aligned}\right.
$$

Consider the function $u^{\varphi}$. Direct computation gives

$$
\nabla u^{\varphi}=u^{\varphi}(\log u \nabla \varphi+\varphi \nabla \log u)
$$

$\Delta u^{\varphi}=u^{\varphi}\left(|\log u \nabla \varphi+\varphi \nabla \log u|^{2}+\log u \Delta \varphi+2 \nabla \varphi \cdot \nabla \log u+\varphi \Delta \log u\right)$

Using Corollary 2.6, we have

$$
|\nabla \log u|=\mathrm{O}\left(e^{(1 / 3-\delta / 2) r}\right)
$$

and

$$
\begin{aligned}
|\log u(x)| & =\left|\log u(x)-\log u\left(p^{\prime}\right)\right| \\
& \leq \int_{\gamma}|\nabla \log u| \\
& \leq \sup _{B_{p}(r(x))}|\nabla \log u| \cdot d\left(x, p^{\prime}\right) \\
& \leq \tilde{C} e^{(1 / 3-\delta / 2) r(x)}(r+1) \\
& =\mathrm{O}\left(e^{r / 3}\right),
\end{aligned}
$$


where $\gamma$ is the geodesic segment connecting $x$ and $p^{\prime}$.

Observe that

$$
\Delta \log u=\frac{\Delta u}{u}-\frac{|\nabla u|^{2}}{u^{2}}=-|\nabla \log u|^{2}
$$

Therefore

$$
\Delta u^{\varphi} \leq u^{\varphi}\left(C_{1} e^{(-1 / 3-\delta / 2) r}+\left(\varphi-\varphi^{2}\right)|\nabla \log u|^{2}\right) .
$$

Let $\psi \in C^{\infty}(\mathbb{R})$ be a function such that

$$
\left\{\begin{array}{l}
1 \leq \psi \leq C_{2}, \\
\psi^{\prime}(t)=-\frac{1}{|t| \log ^{2}|t|} \text { for }|t| \geq R_{1}, \\
-\psi^{\prime}(t) \geq 12\left|\psi^{\prime \prime}(t)\right| \geq 0 \text { for for all } t .
\end{array}\right.
$$

Such a function could be constructed by elementary calculas.

Let $\xi=\log u$. Set

$$
F(x)=\psi\left(\xi(x)-e^{-\beta r(x)}\right) \cdot u^{\varphi},
$$

where $\beta$ is a positive number to be determined later. We have for $\psi=$ $\psi\left(\xi(x)-e^{-\beta r(x)}\right)$

$$
\nabla \psi=\psi^{\prime} \cdot\left(\nabla \xi+\beta e^{-\beta r} \nabla r\right)
$$

$$
\Delta \psi=\psi^{\prime \prime} \cdot\left|\nabla \xi+\beta e^{-\beta r} \nabla r\right|^{2}+\psi^{\prime} \cdot\left(-|\nabla \xi|^{2}+\beta e^{-\beta r}\left(\beta \Delta r-\beta^{2}\right)\right) .
$$


Therefore

$$
\begin{aligned}
\Delta F= & \psi \Delta u^{\varphi}+\Delta \psi u^{\varphi}+2 \nabla \psi \cdot \nabla u^{\varphi} \\
= & \psi \Delta u^{\varphi}+\psi^{\prime \prime} u^{\varphi} \cdot\left|\nabla \xi+\beta e^{-\beta r} \nabla r\right|^{2} \\
& +\psi^{\prime} u^{\varphi} \cdot\left((2 \varphi-1)|\nabla \xi|^{2}+2 \xi \nabla \varphi \cdot \nabla \xi+2 \beta e^{-\beta r} \xi \nabla \varphi \cdot \nabla r\right. \\
& \left.+2 \beta \varphi e^{-\beta r} \nabla \xi \cdot \nabla r+e^{-\beta r}\left(\beta \Delta r-\beta^{2}\right)\right) .
\end{aligned}
$$

Using (2.6) (2.7) and (2.8), we obtain the following estimate

$$
\begin{aligned}
\Delta F \leq & u^{\varphi}\left[C_{1} C_{2} \psi e^{(-1 / 3-\delta / 2) r}+2 \beta^{2} e^{-2 \beta r}\left|\psi^{\prime \prime}\right|+\psi^{\prime} \cdot\left(-C_{3} e^{(-1 / 3-\delta / 2) r}\right.\right. \\
& \left.\left.+e^{-\beta r}\left(\beta \Delta r-\beta^{2}-\beta \varphi\right)\right)\right]+u^{\varphi}|\nabla \xi|^{2}\left[2\left|\psi^{\prime \prime}\right|+\left(2 \varphi-1-\beta \varphi e^{-\beta r}\right) \psi^{\prime}\right] \\
\leq & u^{\varphi}\left(C_{4} e^{(-1 / 3-\delta / 2) r}+\psi^{\prime} \cdot\left(\beta \Delta r-\beta^{2}-\beta\right) e^{-\beta r}\right)+u^{\varphi} \psi^{\prime}|\nabla \xi|^{2}\left(\frac{1}{6}-\beta e^{-\beta r}\right) .
\end{aligned}
$$

Here we have used the inequalities $2 / 3 \leq \varphi \leq 1$ and $\nabla \xi \cdot \nabla r \leq|\nabla \xi|^{2}+1$.

Since $\Delta r \geq n-1$, we can take $\beta<\delta / 4$ sufficiently small so that

$$
\left(\beta \Delta r-\beta^{2}-\beta\right) e^{-\beta r} \geq C_{5} e^{-\beta r}
$$

At points $x$ such that $\xi=\xi(x) \geq R_{1}+1$, from (2.7) we have

$$
\xi=\mathrm{o}\left(e^{r / 3}\right) \text { and } \log \xi=\mathrm{O}(r)
$$


Together with the definition of $\psi$, this yields

$$
\begin{aligned}
\psi^{\prime} \cdot\left(\beta \Delta r-\beta^{2}-2 \beta\right) e^{-\beta r} & \leq-\frac{1}{|\xi| \log ^{2}|\xi|} C_{5} e^{-\beta r} \\
& \leq-C_{6} \frac{1}{r^{2}} e^{-(1 / 3+\beta) r} \\
& \leq-C_{7} e^{-(1 / 3+\delta / 4) r}
\end{aligned}
$$

for $r$ sufficiently large. Here we have used that $e^{-(1 / 3+\beta) r} / r^{2}=O\left(e^{-(1 / 3+\delta / 4) r}\right)$ since $\beta<\delta / 4$.

It follows that for $\xi \geq R_{1}+1$, we have

$$
\begin{aligned}
\Delta F \leq & u^{\varphi}\left(C_{4} e^{(-1 / 3-\delta / 2) r}-C_{7} e^{-(1 / 3+\delta / 4) r}\right) \\
& +u^{\varphi} \psi^{\prime}|\nabla \xi|^{2}\left(\frac{1}{6}-2 \beta e^{-\beta r}\right) .
\end{aligned}
$$

If $R_{2}$ is sufficiently large then $C_{4} e^{(-1 / 3-\delta / 2) r}-C_{7} e^{-(1 / 3+\delta / 4) r}<0$ and $\frac{1}{6}-$ $2 \beta e^{-\beta r}>0$ for $r \geq R_{2}$, so that $\Delta F \leq 0$ on $T\left(\theta_{1}, R_{2}\right)$.

The remaining case is when $\xi \leq R_{1}+1$. We then have $u=e^{\xi} \leq C_{8}=$ $e^{R_{1}+1}$ is bounded. In addition, $u^{\varphi} \leq C_{8} u^{1 / 2}$ and $u^{\varphi}|\xi| \leq C_{9} u^{1 / 2}$. Using the fact that $|\psi|,\left|\psi^{\prime}\right|$ and $\left|\psi^{\prime \prime}\right|$ are all bounded, we conclude that

$$
\Delta F \leq C_{10} u^{1 / 2}|\nabla \xi|^{2}+C_{11} e^{-\beta r}
$$

Define

$$
G=F+C_{12} u^{1 / 2}+e^{-\alpha_{1} r} .
$$


This is a positive function with $\alpha_{1}<\beta$ and $C_{11}$ to be determined. It is clear that $G_{F}=C_{12} u^{1 / 2}+e^{-\alpha_{1} r}$ is superharmonic. Therefore

$$
\begin{aligned}
\Delta G & =\Delta F+C_{12} u^{1 / 2}\left(-\frac{1}{4}|\nabla \xi|^{2}\right)+e^{-\alpha r}\left(\alpha^{2}-\alpha \Delta r\right) \\
& \leq C_{10} u^{1 / 2}|\nabla \xi|^{2}+C_{11} e^{-\beta r}-\frac{C_{12}}{4} u^{1 / 2}|\nabla \xi|^{2}-C_{13} e^{-\alpha r} \\
& =\left(C_{10}-\frac{C_{12}}{4}\right) u^{1 / 2}|\nabla \xi|^{2}+C_{11} e^{-\beta r}-C_{13} e^{-\alpha r} \\
& \leq 0
\end{aligned}
$$

on $T\left(\theta_{1}, R_{3}\right)$ if $C_{12}, C_{13}$ and $R_{3}$ are sufficiently large.

Combine (2.11), (2.13) and the superharmonicity of $G-F$, we have for all $\xi$,

$$
\Delta G \leq 0
$$

on $T\left(\theta_{1}, R_{0}\right)$ with $R_{0}=\max \left(R_{2}, R_{3}\right)$.

Since $u$ is harmonic,

$$
\Delta\left(C_{14} G-u\right) \leq 0
$$

on $T\left(\theta_{1}, R_{0}\right)$, where $C_{14}>1$ is a constant to be determined. Observe that $F=\psi u \geq u$ on $\partial C_{p}\left(\theta_{1}\right)$. Therefore

$$
C_{14} G-u \geq C_{14} F-u \geq 0
$$


on $\partial C\left(\theta_{1}\right) \backslash B_{p}\left(R_{3}\right)$. If $C_{14}$ is sufficiently large, we also have

$$
C_{14} G-u \geq 0
$$

on $\partial B_{p}\left(R_{3}\right) \cap C\left(\theta_{1}\right)$. By the maximum principle,

$$
C_{14} G \geq u
$$

on $T\left(\theta_{1}, R_{0}\right)$. In particular, on $T\left(\theta_{3}, R_{0}\right)$ we have

$$
\begin{aligned}
u \leq C_{14} G & =C_{14} \psi u^{2 / 3}+C_{12} C_{14} u^{1 / 2}+e^{-\alpha_{1} r} \\
& \leq C_{1} C_{14} u^{2 / 3}+C_{9} C_{10} u^{1 / 2}+e^{-\alpha_{1} r}
\end{aligned}
$$

which implies that $u$ is bounded on $T\left(\theta_{3}, R_{0}\right)$. By the gradient estimate $u$ is also bounded on $\overline{B_{p}\left(R_{0}\right)}$. Therefore, positive harmonic functions on $C_{p}\left(\theta_{1}\right)$ which vanish on $\overline{C_{p}\left(\theta_{1}\right)} \cap S(\infty)$ are uniformly bounded on $\overline{C_{p}\left(\theta_{3}\right)}=$ $\overline{T\left(\theta_{3}, R_{0}\right)} \cup \overline{B_{p}\left(R_{0}\right)}$. Now applying Lemma 2.7 we have for all $x \in T\left(\theta_{2}, R_{0}\right)$,

$$
u(x) \leq \tilde{C} e^{-\alpha r(x)} u\left(p^{\prime}\right) .
$$

The truncated cones $T\left(\theta_{2}, R_{0}\right)$ and $T\left(\theta_{2}, 1\right)$ differ by a precompact set, it then follows from the gradient estimate that the Harnack inequality above actually holds on $T\left(\theta_{2}, 1\right)$. This completes the proof of Theorem 2.2.

Proof of Theorem 2.3. Without loss of generality we only need to prove The- 
orem 2.3 under the condition $u\left(p^{\prime}\right)=v\left(p^{\prime}\right)=1$.

By Theorem 2.2, we have

$$
u, v \leq C_{1} e^{-\alpha_{1} r}
$$

Let $\xi=-\log u$. From the gradient estimate we have

$$
\nabla \xi=\mathrm{O}\left(e^{(1 / 3-\delta / 2) r}\right)
$$

and

$$
\xi=\mathrm{o}\left(e^{r / 3}\right)
$$

Thus we have

$$
C_{2} r \leq \xi \leq C_{3} e^{r / 3}
$$

on $T\left(\theta_{1}, R_{1}\right)$. It follows from (2.14) and (2.16) that

$$
\xi^{-\epsilon} \geq e^{-\epsilon r / 3} \geq C_{4} v
$$

for $\epsilon>0$ sufficiently small.

We will construct a function $F \in C^{\infty}\left(C_{p}\left(\theta_{1}\right)\right)$ satisfying

$$
\begin{gathered}
\text { (i) } \Delta F \leq 0 \text { on } T\left(\theta_{1}, R_{0}\right), \\
\text { (ii) } F \geq v \text { on } \partial T\left(\theta_{1}, R_{0}\right), \\
\text { (iii) } F \leq C_{5} u \text { on } T\left(\theta_{2}, R_{0}\right) \text {. }
\end{gathered}
$$

It will then follow from the maximum principle that $v \leq F$ on $T\left(\theta_{1}, R_{0}\right)$. In 
particular, $v \leq C_{5} u$ on $T\left(\theta_{2}, R_{0}\right)$, which gives the first inequality in Theorem

2.3. By exchanging $u$ and $v$ we get the second inequality immediately.

We now proceed to construct $F$ satisfying (i),(ii) and (iii). By Lemma 2.4, there exists $\varphi \in C^{\infty}(M)$ with $0 \leq \varphi \leq 1$ such that

$$
\left\{\begin{aligned}
\varphi & =0 \text { on } T\left(\theta_{2}, R_{1}\right) \\
\varphi & =1 \text { on } \partial C_{p}\left(\theta_{1}\right) \backslash B_{p}\left(R_{1}\right) \\
|\nabla \varphi| & =\mathrm{O}\left(e^{-r}\right) \text { on } T\left(\theta_{1}, R_{1}\right) \\
|\Delta \varphi| & =\mathrm{O}\left(e^{-(2 / 3+\delta) r}\right) \text { on } T\left(\theta_{1}, R_{1}\right)
\end{aligned}\right.
$$

Consider the function $f=u^{1-\varphi} \xi^{-\epsilon \varphi}$. We have

$$
\begin{aligned}
& f=u \text { on } T\left(\theta_{2}, R_{1}\right) \\
& f=\xi^{-\epsilon} \geq C_{4} v \text { on } \partial C_{p}\left(\theta_{1}\right) \backslash B_{p}\left(R_{1}\right)
\end{aligned}
$$

and

$$
\nabla f=f \cdot(\xi \nabla \varphi-(1-\varphi) \nabla \xi-\epsilon \log \xi \nabla \varphi-\epsilon \varphi \nabla \log \xi)
$$

$$
\begin{aligned}
\Delta f= & f \cdot\left(|\xi \nabla \varphi-(1-\varphi) \nabla \xi-\epsilon \log \xi \nabla \varphi-\epsilon \varphi \nabla \log \xi|^{2}\right. \\
& +\xi \Delta \varphi+2 \nabla \varphi \cdot \nabla \xi-(1-\varphi) \Delta \xi-\epsilon \log \xi \Delta \varphi-2 \epsilon \nabla \log \xi \cdot \nabla \varphi-\epsilon \varphi \Delta \log \xi) .
\end{aligned}
$$

Observe that

$$
\Delta \xi=-\frac{\Delta u}{u}+|\nabla \xi|^{2}=|\nabla \xi|^{2}
$$


and

$$
\Delta \log \xi=\frac{\Delta \xi}{\xi}-\frac{|\nabla \xi|^{2}}{\xi^{2}}=\frac{|\nabla \xi|^{2}}{\xi}-|\nabla \log \xi|^{2}
$$

Therefore by (2.15), (2.17) and (2.19) we have

$\Delta f \leq f \cdot\left[\left(\varphi^{2}-\varphi\right)|\nabla \xi|^{2}+\epsilon \varphi(1-2 \varphi) \frac{|\nabla \xi|^{2}}{\xi}+\left(\epsilon^{2} \varphi^{2}+\epsilon \varphi\right)|\nabla \log \xi|^{2}+C_{6} e^{-(1 / 3+\delta / 2) r}\right]$

Let $\psi \in C^{\infty}(\mathbb{R})$ be a function such that

$$
\left\{\begin{array}{l}
1 \leq \psi \leq C_{7} \\
\psi^{\prime}(t)=\frac{1}{|t| \log ^{2}|t|} \text { for }|t| \geq R_{2}, \\
\psi^{\prime}(t) \geq 12\left|\psi^{\prime \prime}(t)\right| \geq 0 \text { for for all } t .
\end{array}\right.
$$

Set

$$
F(x)=\psi\left(\xi+e^{-\beta r(x)}\right) \cdot f
$$

We have for $\psi=\psi\left(\xi+e^{-\beta r(x)}\right)$,

$$
\nabla \psi=\psi^{\prime} \cdot\left(\nabla \xi-\beta e^{-\beta r} \nabla r\right)
$$

$$
\Delta \psi=\psi^{\prime \prime} \cdot\left|\nabla \xi-\beta e^{-\beta r} \nabla r\right|^{2}+\psi^{\prime} \cdot\left(\Delta \xi+e^{-\beta r}\left(\beta^{2}-\beta \Delta r\right)\right)
$$


Then we have

$$
\begin{aligned}
\Delta F= & \psi \Delta f+\Delta \psi f+2 \nabla \psi \cdot \nabla f \\
= & \psi \Delta f+\psi^{\prime \prime} f \cdot\left|\nabla \xi-\beta e^{-\beta r} \nabla r\right|^{2} \\
& +\psi^{\prime} f \cdot\left(-|\nabla \xi|^{2}+e^{-\beta r}\left(\beta^{2}-\beta \Delta r\right)\right)+2 \psi^{\prime} \cdot\left(\nabla \xi-\beta e^{-\beta r} \nabla r\right) \cdot \nabla f \\
\leq & \psi f\left[\left(\varphi^{2}-\varphi\right)|\nabla \xi|^{2}+\epsilon \varphi(1-2 \varphi) \frac{|\nabla \xi|^{2}}{\xi}+\left(\epsilon^{2} \varphi^{2}+\epsilon \varphi\right)|\nabla \log \xi|^{2}\right. \\
& \left.+C_{6} e^{-(1 / 3+\delta / 2) r}\right]+\psi^{\prime} f\left[(2 \varphi-3)|\nabla \xi|^{2}-2 \epsilon \varphi \frac{|\nabla \xi|^{2}}{\xi}+2 \beta(1-\varphi) e^{-\beta r} \nabla \xi \cdot \nabla r\right. \\
& \left.+2 \epsilon \beta \varphi e^{-\beta r} \nabla \log \xi \cdot \nabla r+C_{8} e^{-\beta r}\left(\beta^{2}-\beta \Delta r\right)\right] \\
\leq & \psi f\left[\varphi(\varphi-1)|\nabla \xi|^{2}+\varphi(\epsilon-2 \epsilon \varphi) \frac{|\nabla \xi|^{2}}{\xi}+\varphi\left(\epsilon^{2} \varphi+\epsilon\right)|\nabla \log \xi|^{2}\right. \\
& \left.+C_{6} e^{-(1 / 3+\delta / 2) r}\right]+\psi^{\prime} f\left[(2 \varphi-3+2 \beta(1-\varphi+\epsilon \varphi))|\nabla \xi|^{2}\right. \\
& \left.+C_{9} e^{-2 \beta r}+C_{8} e^{-\beta r}\left(\beta^{2}-\beta \Delta r\right)\right] .
\end{aligned}
$$

Here we have used the inequalities $e^{-\beta r} \nabla \xi \cdot \nabla r \leq|\nabla \xi|^{2}+e^{-2 \beta r}$ and $|\nabla \xi|^{2} / \xi \leq$ $|\nabla \xi|^{2}$

As in the proof of Theorem 2.2 , we can choose $\beta<\delta / 4$ to be sufficiently small and $R_{0}$ sufficiently large so that

$$
\begin{aligned}
\Delta F & \leq C_{6} C_{7} e^{-(1 / 3+\delta / 2) r}+C_{10} e^{-\beta r} /\left(\xi \log ^{2} \xi\right) \\
& \leq C_{6} C_{7} e^{-(1 / 3+\delta / 2) r}+C_{11} e^{-(1 / 3+\delta / 4) r} \\
& \leq 0
\end{aligned}
$$


on $T\left(\theta_{1}, R_{0}\right)$. This is possible because $\xi=\mathrm{o}\left(e^{r / 3}\right)$ and $\log \xi=\mathrm{o}(r)$.

We already know that

$$
F=\psi f \geq C_{4} v
$$

on $\partial C_{p}\left(\theta_{1}\right) \backslash B_{p}\left(R_{0}\right)$. Therefore $C_{12} F \geq v$ on $\partial T\left(\theta_{1}, R_{0}\right)$ if $C_{12}$ is sufficiently large. Since $v$ is harmonic, by the maximum principle, we have $C_{12} F \geq v$ on $\overline{T\left(\theta_{1}, R_{0}\right)}$. In particular,

$$
v \leq C_{12} F \leq C_{7} C_{12} u
$$

on $\overline{T\left(\theta_{2}, R_{0}\right)}$.

Since $u\left(p^{\prime}\right)=v\left(p^{\prime}\right)=1$, by the gradient estimate we have

$$
C_{13} \leq u, v \leq C_{14}
$$

on $B_{p}\left(R_{0}\right) \cap T\left(\theta_{2}, 1\right)$. Then $\frac{u}{v} \geq \tilde{C}^{-1}$ on $T\left(\theta_{2}, 1\right)$ with $\tilde{C}=\max \left(\frac{C_{13}}{C_{14}}, C_{7} C_{12}\right)$.

Remark 2.1 As remarked in Section 2.1, Theorem 0.4 follows immediately from Theorem 2.2 and Theorem 2.3. 


\section{Bibliography}

[And83] Michael T. Anderson, The Dirichlet problem at infinity for manifolds of negative curvature, J. Differential Geom. 18 (1983), no. 4, 701721 (1984).

[AS85] Michael T. Anderson and Richard Schoen, Positive harmonic functions on complete manifolds of negative curvature, Ann. of Math. (2) 121 (1985), no. 3, 429-461.

[Bor92] Albert Borbély, A note on the Dirichlet problem at infinity for manifolds of negative curvature, Proc. Amer. Math. Soc. 114 (1992), no. 3, 865-872.

[Cho84] Hyeong In Choi, Asymptotic Dirichlet problems for harmonic functions on Riemannian manifolds, Trans. Amer. Math. Soc. 281 (1984), no. 2, 691-716.

[Hsu03] Elton P. Hsu, Brownian motion and Dirichlet problems at infinity, Ann. Probab. 31 (2003), no. 3, 1305-1319.

[Pet06] Peter Petersen, Riemannian geometry, second ed., Graduate Texts in Mathematics, vol. 171, Springer, New York, 2006.

[Sul83] Dennis Sullivan, The Dirichlet problem at infinity for a negatively curved manifold, J. Differential Geom. 18 (1983), no. 4, 723-732 (1984).

[SY94] R. Schoen and S.-T. Yau, Lectures on differential geometry, Conference Proceedings and Lecture Notes in Geometry and Topology, I, International Press, Cambridge, MA, 1994, Lecture notes prepared by Wei Yue Ding, Kung Ching Chang [Gong Qing Zhang], Jia Qing Zhong 
and Yi Chao Xu, Translated from the Chinese by Ding and S. Y. Cheng, Preface translated from the Chinese by Kaising Tso.

[Yau75] Shing Tung Yau, Harmonic functions on complete Riemannian manifolds, Comm. Pure Appl. Math. 28 (1975), 201-228. 